\title{
Journal of Fluid Mechanics
}

http://journals.cambridge.org/FLM

Additional services for Journal of Fluid Mechanics:

Journal of

Fluid Mechanies

Email alerts: $\underline{\text { Click here }}$

Subscriptions: Click here

Commercial reprints: Click here

Terms of use : $\underline{\text { Click here }}$

\section{Vortex scattering by step topography}

A. K. HINDS, E. R. JOHNSON and N. R. MCDONALD

Journal of Fluid Mechanics / Volume 571 / January 2007, pp 495 - 505

DOI: 10.1017/S002211200600348X, Published online: 04 January 2007

Link to this article: http://journals.cambridge.org/abstract S002211200600348X

How to cite this article:

A. K. HINDS, E. R. JOHNSON and N. R. MCDONALD (2007). Vortex scattering by step topography. Journal of Fluid Mechanics, 571, pp 495-505 doi:10.1017/S002211200600348X

Request Permissions : $\underline{\text { Click here }}$ 


\title{
Vortex scattering by step topography
}

\author{
By A. K. HINDS, E. R. JOHNSON AND N. R. MCDONALD
}

Department of Mathematics, University College London, Gower Street, London, WC1E 6BT, UK

(Received 23 August 2006 and in revised form 4 October 2006)

The scattering at a rectilinear step change in depth of a shallow-water vortex pair consisting of two patches of equal but opposite-signed vorticity is studied. Using the constants of motion, an explicit relationship is derived relating the angle of incidence to the refracted angle after crossing. A pair colliding with a step from deep water crosses the escarpment and subsequently propagates in shallow water refracted towards the normal to the escarpment. A pair colliding with a step from shallow water either crosses and propagates in deep water refracted away from the normal or, does not cross the step and is instead totally internally reflected by the escarpment. For large depth changes, numerical computations show that the coherence of the vortex pair is lost on encountering the escarpment.

\section{Introduction}

The ubiquity of vortices in the surf-zone has been revealed by several numerical and observational studies. Peregrine (1998) shows that vortices frequently 'pair-up', forming dipoles which subsequently propagate for significant periods. It has been shown (Gorshkov, Ostrovsky \& Soustova 2000) that dipoles are robust vortical distributions, able to survive for many rotation periods. The durability of dipoles in the surf zone has been demonstrated numerically by Özkan-Haller \& Kirby (1999), Slinn et al. (1998), Chen et al. (1999), Bühler \& Jacobson (2001), and through field observations by Smith \& Largier (1995).

There is evidence that surf-zone vortices are likely to be significantly affected by beach morphology. Richardson (2000) finds, in the limit of small radii compared to typical vortex separation and to the length scale for variations in depth, that monopolar vortices move along isobaths at a speed proportional to the logarithm of the depth gradient. Bühler \& Jacobson (2001) present a theoretical and numerical study for waves and vortices in a shallow-water model with bottom topography. The evolution of dipole structures in the surf-zone is studied using a simple method to represent the effect of a sloping beach and invoking the conservation of potential vorticity. It is found that dipole separation decreases on moving into deeper water, and increases on moving into shallower water.

Perhaps the simplest choice of beach topography is the rectilinear step. Johnson \& McDonald (2004) consider two-dimensional finite-area uniform vortex patches (with equal and opposite circulation) normally incident on a rectilinear step of arbitrary height. They show that a vortex pair starting in relatively deep water, crosses the escarpment and translates in relatively shallow water with increased centroid separation. The change in centroid separation is a consequence of the strong interaction between the vortex and its oppositely signed image (in the step topography) as the pair approaches the escarpment. Similarly, a pair starting in shallow water, crosses the escarpment and subsequently translates in deep water with decreased 


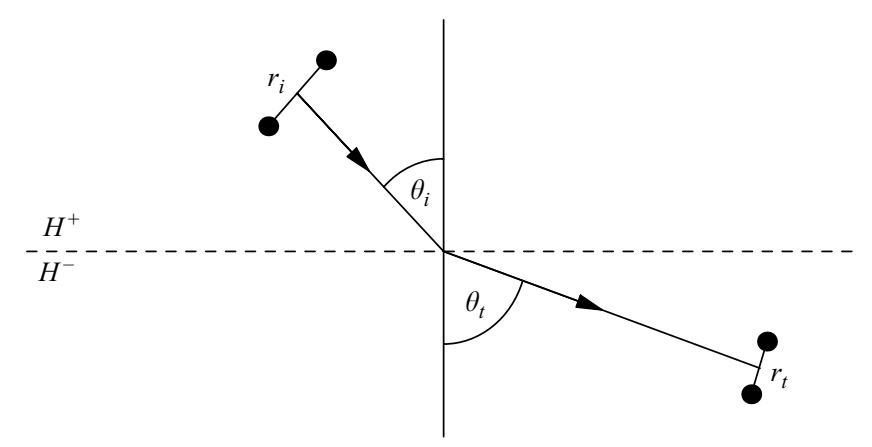

FIGURE 1. Sketch of a vortex pair crossing a step (along $y=0$, indicated by the dashed line). The subscripts $i$ and $t$ denote the quantities before and after crossing the step, i.e. 'incident' and 'transmitted'. The centroid separation is denoted $r$ with the normal angle to the step $\theta$.

centroid separation. In this case, the vortex is influenced by a like-signed image in the step.

The present work considers a vortex pair translating at an arbitrary angle of incidence to the step (figure 1). Attention is confined to the scattering of two equalarea, two-dimensional finite-area vortex patches with opposite sign by a rectilinear step. The conservation of energy, circulation, volume and linear impulse determines the trajectory of the pair after a collision with the escarpment, provided there is minimal shedding and the shape of each patch remains close to circular.

A pair crossing from deep to shallow water is refracted towards the normal, and similarly a pair translating from shallow water to deep water refracts away from the normal. Hence, it is expected there exists a critical angle for a pair crossing from shallow to deep water in which the refracted trajectory on the deep side is parallel to the step. For angles of incidence greater than this critical angle, the pair no longer crosses the step and is instead reflected by the step. This scattering behaviour of vortex pairs is analogous to light undergoing total internal reflection when incident upon a medium of lower refractive index.

Section 2 outlines the mathematical formulation of the problem and derives an explicit relationship determining the angle of reflection given the initial separation of the pair, the angle of incidence and the size of the vortex patches comprising the pair. In $\S 3$, the evolution of such pairs is computed numerically using an adapted contour surgery algorithm based on Dritschel (1988), for various angles of incidence from both shallow and deep water. These computation results are then compared to that predicted by the relationship of $\S 2$. Section 4 presents conclusions.

\section{Analytical formulation}

The two-dimensional flow of a shallow inviscid fluid with a rigid lid above finiteheight topography is governed by the conservation of potential vorticity, $q=\omega / H$,

$$
q_{t}+\boldsymbol{u} \cdot \nabla q=0
$$

where $H$ is the local fluid depth and $\omega=v_{x}-u_{y}$ is the vertical component of the vorticity. The total kinetic energy, $\mathscr{E}$,

$$
\mathscr{E}=\int \psi \omega \mathrm{d} x \mathrm{~d} y,
$$


is a constant of the motion and $\psi$ is a volume flux streamfunction giving velocity components $(u, v)=H^{-1}\left(-\psi_{y}, \psi_{x}\right)$. Here and below, $\omega$ is taken to decay sufficiently rapidly at infinity to give convergent integrals. For topography that varies in one direction only (the $y$ direction, say), the linear impulse

$$
\mathscr{I}=\int Y(y) \omega \mathrm{d} x \mathrm{~d} y,
$$

where $Y(y)=\int^{y} H\left(y^{\prime}\right) \mathrm{d} y^{\prime}$ gives a second constant of the motion (Johnson, Hinds $\&$ McDonald 2005). This paper considers vortex pairs consisting of two finite-area equal-volume patches of oppositely signed uniform vorticity, where the volume

$$
\mathscr{V}=\int H(y) \mathrm{d} x \mathrm{~d} y,
$$

of each patch (with the integral taken over the support of either patch) remains constant by mass conservation, giving a third constant of motion. Conservation of $q$ and $\mathscr{V}$ together imply the circulations $\pm \Gamma$ of the patches are individually conserved, where

$$
\Gamma=\int \omega \mathrm{d} x \mathrm{~d} y>0,
$$

with the integral taken over the positively signed patch only.

The energy of a vortex pair consisting of circular patches of uniform vorticity, centred at $\left(x_{1}, y_{1}\right)$ and $\left(x_{2}, y_{2}\right)$ near a finite escarpment with depth $H^{+}$in $y>0$ and $H^{-}$in $y<0$ when the pair translates in deeper water is (Johnson \& McDonald 2004)

$$
\mathscr{E}=\frac{\Gamma^{2} H^{+}}{2 \pi}\left\{\log \left(\frac{r}{a}\left|\frac{2\left(y_{1} y_{2}\right)^{1 / 2}}{r^{\prime}}\right|^{\alpha}\right)+\frac{1}{4}\right\},
$$

where $\gamma=H^{-} / H^{+}$is the depth ratio, $\alpha=(\gamma-1) /(\gamma+1)$ the image strength due to the topography, $a$ the patch radius, $r$ the vortex pair centroid separation and $r^{\prime}=\left[\left(x_{1}-x_{2}\right)^{2}+\left(y_{1}+y_{2}\right)^{2}\right]^{1 / 2}$. The depth ratio lies in the range $0 \leqslant \gamma<\infty$, so the image strength satisfies $-1 \leqslant \alpha \leqslant 1$. For a pair far from escarpment, the topographic image terms are negligible, thus $\left(y_{1} y_{2}\right)^{1 / 2} \approx y$ and $r^{\prime} \approx 2 y$, where $y$ is the normal distance from the escarpment to the centre of vorticity of the vortex pair. Then the energy of a pair in deep water $(y>0)$ is

$$
\mathscr{E}=\frac{\Gamma^{2} H^{+}}{2 \pi}\left(\log \chi+\frac{1}{4}\right),
$$

for $\chi=r / a$, the ratio of centroid separation to patch radius. Conservation of circulation, with (2.7) and its equivalent form in $y<0$ where $H^{-}$replaces $H^{+}$, gives the relation between pair separation either side of the step

$$
\chi_{t}=\chi_{i}^{1 / \gamma} \exp [(1 / \gamma-1) / 4],
$$

where subscript $i$ denotes a quantity incident on the step and $t$ denotes the transmitted quantity, assuming the pair has crossed the escarpment. For $\gamma<1$ (i.e. a pair crossing from deep to shallow water), the patch separation increases after crossing and for $\gamma>1$, the separation decreases. The vortices are assumed to remain circular after crossing the step so the centroid separation must be at least twice the radius of the vortices comprising the pair, i.e. $\chi_{t} \geqslant 2$. For each $\chi_{i}$, there is a sufficiently large increase in depth for which $\chi_{t}=2$. This critical depth ratio $\gamma_{d}>1$ (where subscript $d$ 


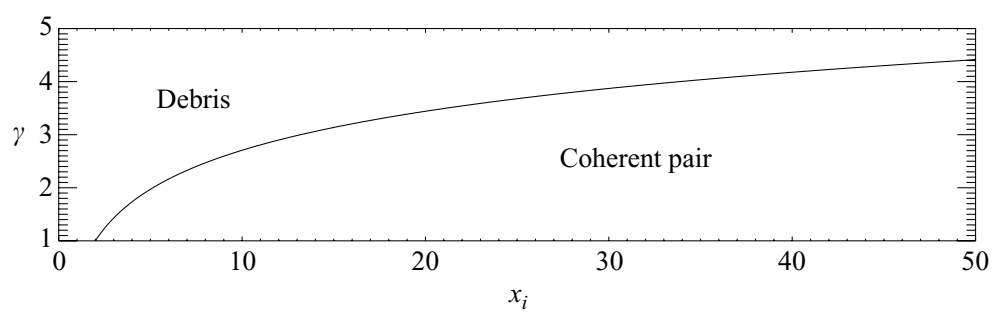

FIGURE 2. The evolution regimes determined by the depth ratio $\gamma$ and the initial vortex separation $\chi_{i}$. 'Coherent pair': a pair incident to a step will cross with the vortices in the pair remaining coherent. 'Debris': where significant vortex shedding will occur upon crossing. The dividing curve is the critical depth ratio $\gamma_{d}$, from (2.9).

denotes debris) follows from (2.8) as

$$
\gamma_{d}=\frac{\log \chi_{i}+1 / 4}{\log 2+1 / 4} .
$$

For depth ratios larger than $\gamma_{d}$, the numerical integrations (in $\S 3$ and Johnson \& McDonald 2004) show that the vortices are pulled so close together that they shed vorticity. This region of parameter space is described here as the debris region (figure 2).

Constancy of $\mathscr{V}$, from (2.4), gives $2 H^{+} \pi a_{i}^{2}=2 H^{-} \pi a_{t}^{2}$, and so the incident and transmitted radii are related by

Conservation of linear impulse, $\mathscr{I}$, gives

$$
a_{i}=\sqrt{\gamma} a_{t}
$$

$$
\mathscr{I}=\Gamma H^{+}\left(-y_{1 i}+y_{2 i}\right)=\Gamma H^{-}\left(-y_{1 t}+y_{2 t}\right),
$$

relating the vortex separation perpendicular to the step before and after crossing. In terms of the quantities $r_{i, t}$ and $\theta_{i, t}$ defined in figure 1, (2.11) becomes

$$
r_{i} \sin \theta_{i}=\gamma r_{t} \sin \theta_{t} .
$$

Combining (2.8), (2.10) and (2.12),

$$
\frac{\sin \theta_{i}}{\sin \theta_{t}}=\chi_{i}^{1 / \gamma-1} \sqrt{\gamma} \exp [(1 / \gamma-1) / 4],
$$

an explicit relation between the incident and transmitted angles in terms of the depth ratio $\gamma$ and the incident vortex separation $\chi_{i}$. It follows immediately from (2.13) that there is a depth ratio, $\gamma_{\max }$, where the refracted angle after crossing is a maximum, $\gamma_{\max }=2\left(\log \chi_{i}+1 / 4\right)$. Since $\gamma_{\max }>\gamma_{d}$, a pair crossing from shallow water to deep water at any incident angle cannot reach its maximum refracted angle and importantly never refracts towards the normal as this region of parameter space lies in the debris region.

Figure 3 shows the transmitted angle $\theta_{t}$ as a function of the depth ratio $\gamma$ for fixed incident angle, $\theta_{i}$ and incident vortex separation, $\chi_{i}$. The depth ratio $\gamma$ lies in the range $0 \leqslant \gamma<\infty$ corresponding to a pair incident on a wall $(\gamma=0)$ and a pair incident on a infinitely deep drop-off $(\gamma \rightarrow \infty)$ ). If $\gamma<1$ (so that the pair crosses into relatively shallow water), the pair is refracted towards the normal of the topography (i.e. $\theta_{t}<\theta_{i}$ ). As $\gamma \rightarrow 0$ (so that the topography becomes more 'wall-like'), $\theta_{t} \rightarrow 0$ for all non-zero $\theta_{i}$. In this limit, the vortices forming the pair separate as they approach the step. They subsequently propagate far along the wall as monopoles before crossing where they 


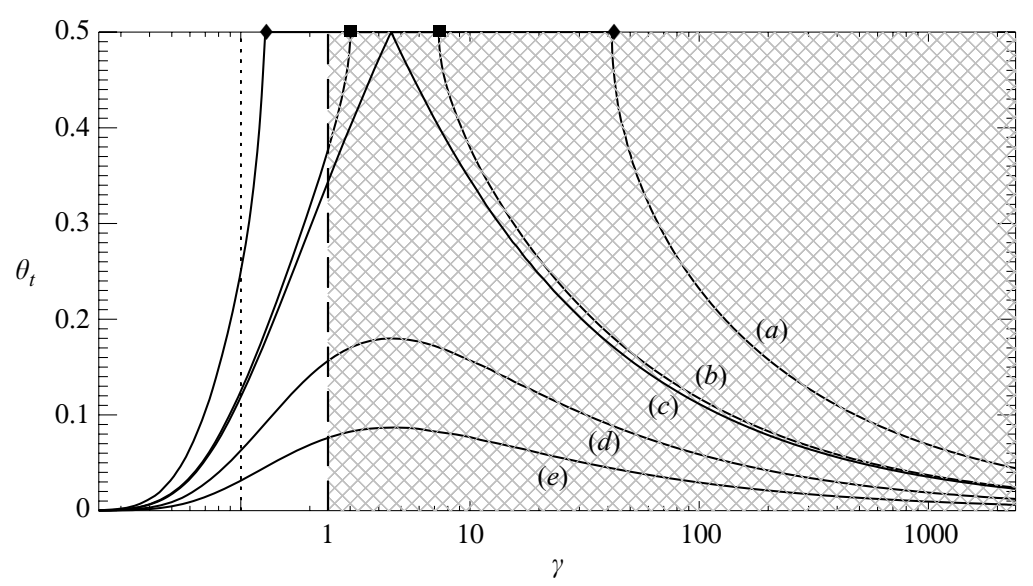

FIGURE 3. The refracted angle (in units of $\pi$ ), $\theta_{t}$ as a function of depth ratio $\gamma$ for vortex separation $\chi=7.5$ at varying angles of incidence $\theta_{i},(a) \theta_{i}=\pi / 4,(b) \theta_{i}=\pi / 8,(c) \theta_{i}=0.119 \pi$, (d) $\theta_{i}=\pi / 16,(e) \theta_{i}=\pi / 32$. The short dashed line is where the step is absent, $\gamma=1$ and $\theta_{i}=\theta_{t}$. The line $\gamma=\gamma_{d}$ is shown long-dashed with the debris region, $\gamma>\gamma_{d}$ hatched. Solid marks show the range of depth ratios where the incident pair can be totally internally reflected.

translate with large separation almost perpendicularly away from the escarpment in shallow water (cf. Johnson \& McDonald 2004). When $\gamma>1$ (so the pair would cross from shallow water into relatively deep water), an incident pair would be refracted away from the normal (as could be deduced from time reversal with the results just noted). However, for certain incident angles, the transmitted angle can be equal to $\pi / 2$. This corresponds to the pair translating parallel to the step after it has crossed. Let this critical incident angle be $\theta_{c}$, so

$$
\sin \theta_{c}=\chi_{i}^{1 / \gamma-1} \sqrt{\gamma} \exp [(1 / \gamma-1) / 4]
$$

For incident angles greater than $\theta_{c}$, with all other parameters fixed, the vortex pair undergoes total internal reflection (TIR): it does not cross the step, but is instead reflected and translates away from the step at its incident angle. Total internal reflection here is analogous to Snell's law for refraction of a light ray as it enters a medium with a different refractive index. The angle of incidence (measured relative to the normal to the interface) decreases when light passes into a medium of higher refractive index and increases passing into a medium of lower refractive index, with deeper water corresponding to a lower refractive index in the present work. It has been established that a pair crossing the step with depth ratio greater than $\gamma_{d}$, given by (2.9), will shed vorticity as it crosses the step. This debris region is cross-hatched in figure 3 and here the pair does not have a well-defined transmitted angle. However, for $\gamma>\gamma_{d}$, the pair can still undergo TIR, as on curve $(b)$ in figure 3 . Here there is a range of depth ratios, $\gamma$ (delimited by solid squares in figure 3 ) where a pair undergoes TIR and remains coherent despite lying inside the debris region. Similarly for curve $(a)$ in figure 3 , solid diamonds mark out where a pair with incident angle $\theta_{i}=\pi / 4$ will perform total internal reflection. For larger depth ratios, a translating pair could exist in the debris region, reflecting without shedding. However, for depth ratios greater than the right-hand diamond, an incident pair is unable to undergo reflection and instead crosses the step and, being in the debris region, undergoes significant vortex shedding. 




FIGURE 4. The refracted angle, $\theta_{t}$ as a function of incident angle, $\theta_{i}$ (both in units of $\pi$ ) with incident separation $\chi_{i}=3$ for different depth ratios, $(i) \gamma=1.3$, (ii) $\gamma=1.2$, (iii) $\gamma=1$. 1 . The dash-dotted line shows where $\theta_{t}=\theta_{i}$, or $\gamma=1$. The marked region shows the debris regime for values of $\gamma$ between $\gamma_{d}$, shown by a long dashed line, up to $\gamma_{\max }$ shown with a dotted line. The shaded region shows where a pair may undergo TIR if the depth ratio is sufficiently large. In the cross-hatched region, the pair always crosses the step shedding vorticity. The contours below the dash-dotted line are the reciprocals of the contours for $\gamma>1$.

Figure 4 shows the transmitted angle, $\theta_{t}$ as a function of incident angle, $\theta_{i}$ with incident separation $\chi_{i}=3$ for different depth ratios. The dash-dotted line shows where $\theta_{t}=\theta_{i}$ and $\gamma=1$ when there is no change of depth. The marked region on each figure shows the debris region for depth ratios up to $\gamma_{\max }$, shown with a dotted line, which is the value of $\gamma$ where the largest $\theta_{t}$ occurs for a given $\theta_{i}$. This region is split into two further regions. In the shaded region shedding occurs unless the depth ratio is large enough so that $\theta_{i}>\theta_{c}$ and the pair undergoes TIR. In the cross-hatched region, the pair crosses leading to vortex shedding.

\section{Numerical computation of vortex-pair scattering}

The instantaneous velocity field for a given vorticity distribution $\omega$ follows from inverting

$$
-\nabla \cdot\left(H^{-1} \nabla \psi\right)=\omega
$$

to obtain the streamfunction $\psi$. For piecewise constant $\omega$, contour surgery (Dritschel 1988) gives a particularly accurate and efficient numerical algorithm for this inversion, allowing contours of potential vorticity to split and merge as dictated by the flow and removing filaments which form during the integrations provided they are dynamically insignificant. Details of the specific algorithm used here, which takes into account finite-height topography and the possibility of a vortex straddling the escarpment can be found in Johnson \& McDonald (2004) and Johnson et al. (2005). The computations here use a resolution with, on average, 150 nodes around a vortex patch (of unit radius), initialized sufficiently far from the step that the effect of the partial images owing to the topography is small (here $r^{\prime} / 2 y \leqslant 1$, cf. (2.7)). The total decrease in vortex patch volume through surgery is less than $1 \%$ unless stated. 


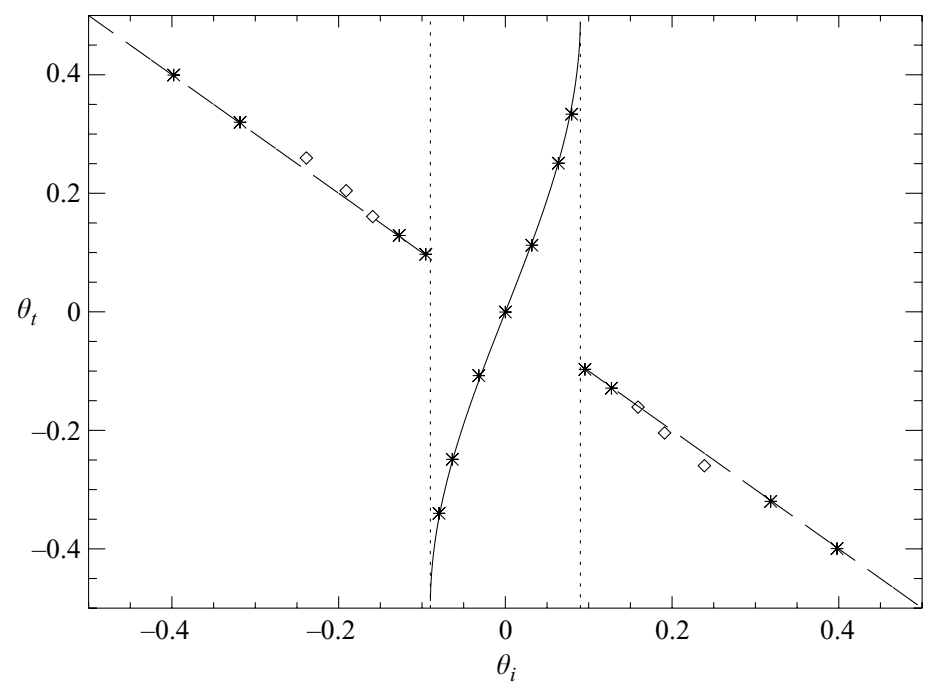

FIGURE 5. Comparison between analytical and numerical results for a vortex patch pair colliding with a step. The transmitted angle $\theta_{t}$ is given as a function of the incident angle $\theta_{i}$ (in units of $\pi$ ) over a step which doubles in depth, $\gamma=2$ and an initial separation $\chi_{i}=20$. The solid line shows where the pair is refracted and the long dashed line shows where the pair undergoes TIR. Vertical dotted lines show where $\theta_{i}=\theta_{c}$. Each marked point plots the incident and refractive angles for a single computation with asterisks showing where shedding is $\leqslant 1 \%$ of the pair's volume, and diamonds showing where vortex shedding is $\geqslant 1 \%$.

\subsection{Refraction at step topography}

Figure 5 compares the theoretical prediction (2.13) for $\theta_{t}$ with numerical results for a vortex pair colliding with a step for various incident angles $\theta_{i}$ for $\chi_{i}=20$ and $\gamma=2$. The solid line is that predicted by (2.13) for a pair being refracted as they cross the step and the dashed line shows where the pair is reflected, i.e. $\theta_{i}>\theta_{c}$. The marked data points give the incident and refracted angles for a given numerical computation. The agreement between the theoretical and computational angles is remarkable when shedding is negligible ( $\leqslant 1 \%$ of the pair volume), as indicated by an asterisk. In this case, there is only a brief time where each vortex of the pair straddles the escarpment, where shedding occurs. Diamonds show where shedding is more significant $(\geqslant 1 \%$, but still small compared with the size of the vortex). Here, vortices spend more time over the step, more vorticity is shed and the agreement, although not as close as for the non-shedding case, is still excellent. Figure 6(a) shows centroid paths for each patch of a pair propagating from deep to shallow water with $\gamma=1 / 2$ and $\left(\theta_{i}, \theta_{t}\right)=(0.222,0.0162)$. The pair is refracted toward the normal after crossing the escarpment into shallow water. As the pair crosses the step, the separation distance between vortex centroids increases under the influence of the oppositely signed images in the step. Figure $6(b)$ shows a pair propagating from shallow to deep water with $\left(\theta_{i}, \theta_{t}\right)=(0.222,0.684)$. In this case, the separation between vortex centroids decreases under the influence of the same-signed images in the step, which now acts as an 'anti-wall' (Johnson \& McDonald 2004).

\subsection{The critical angle and total internal reflection}

Figure 7 shows patch centroid trajectories for a vortex pair propagating from shallow to deep water at the critical angle of incidence so the pair is refracted parallel to the step. In the absence of vortex shedding, the pair would propagate parallel to 
(a)



(b)

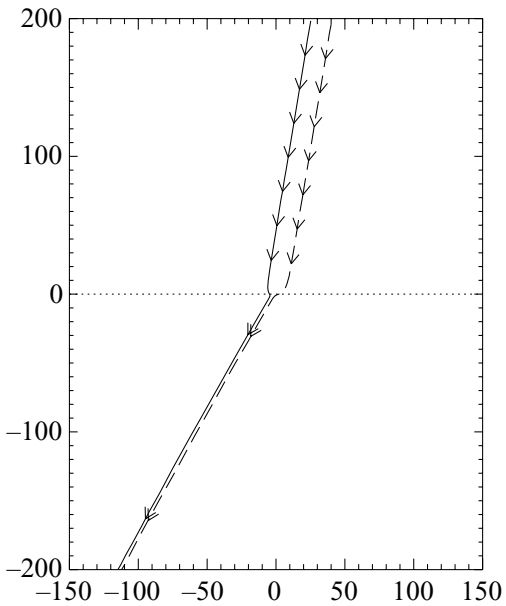

FIGURE 6. Trajectories of patch centroid positions when a pair is refracted by step topography. The initial conditions are the same for each computation, $\theta_{i}=0.222$, with arrows drawn here and hereinafter at equal time intervals. $(a)$ The depth halves and (initially in deep water) the pair crosses the step and translates in shallow water with increased separation and $\theta_{t}=0.684$. (b) The depth doubles and the pair initially in shallow water crosses the step with decreased separation and $\theta_{t}=0.0162$

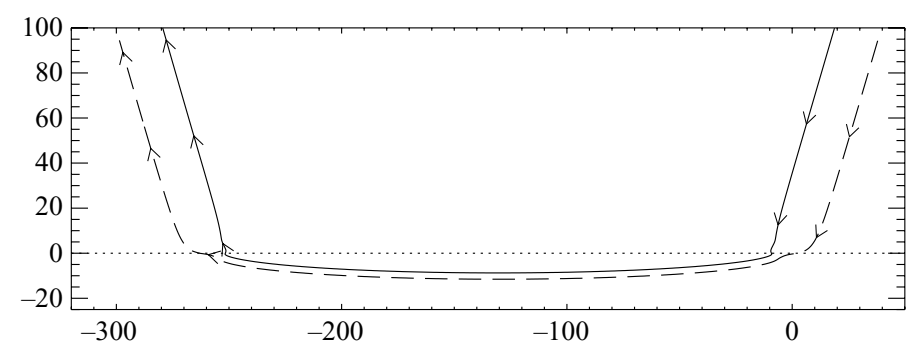

FIGURE 7. Patch centroid trajectories for an incident vortex pair at the critical angle. Weak vortex shedding at the step means one vortex is stronger than the other and the pair eventually recrosses the escarpment, after propagating 300 radius lengths parallel to the step.

the step on the deep side. A small amount of vortex shedding as the pair crosses the escarpment means that the vortices no longer have precisely equal and opposite circulation. The path thus eventually curves back toward the escarpment and the pair crosses back into shallow water. The effect is extremely small: the pair travels nearly 300 radius lengths parallel to the step before it recrosses the escarpment. A similar phenomenon called the Goos-Hähnchen effect occurs in optics: a light beam undergoing TIR propagates a few wavelengths parallel to the interface between different media before being reflected (Yeh 1998). Figure 8(a) shows a trajectory for $\theta_{i}>\theta_{c}$, so the pair undergoes TIR. At no stage do the vortex patches straddle the escarpment and so shedding is minimal. Figure $8(b)$ shows another case of TIR, but with $\theta_{i} \gtrsim \theta_{c}$, so that one of the vortex patches comprising the pair momentarily straddles the escarpment. Since this vortex sheds some vorticity, the vortex pair is no longer comprised of patches with equal and opposite circulation and subsequently the pair 'bounces' along the escarpment. 



FiguRE 8. Trajectories of patch centroids for a vortex pair incident from shallow water. (a) $\theta_{i}>\theta_{c}$, the angle of incidence is greater than the critical angle and the pair is reflected. (b) $\theta_{i} \sim \theta_{c}$, one vortex momentarily straddles the escarpment, shedding vorticity and the pair is captured in relatively shallow water.

\subsection{The debris region}

Figure 9 gives an example where the depth ratio is greater than $\gamma_{d}$, so the trajectories lie in the debris region. The incident angle is chosen so the pair neither reflects nor crosses the escarpment coherently to translate away at a constant transmitted angle. On approaching the step, the pair is forced closer together under the influence of the partial images present from the step. The pair crosses the step and, since $\gamma>\gamma_{d}$ so $\chi_{t}<2$, the pair sheds vorticity leaving one patch with larger (in magnitude) circulation than the other. The subsequent pair trajectory, figure $9(a)$, is almost circular and the pair eventually recrosses the step and, in the shallow region, the pair follows a curved path with much larger radius (figure $9 b$ ). The pair cross the step for the third time into deeper water where, after more shedding, the paths follow tighter circular paths, with a drift parallel to the escarpment.

\section{Conclusions}

The conservation of potential vorticity, energy, linear impulse and mass give an explicit relation for the angle of refraction and separation of vortex centroids for a vortex pair colliding with a rectilinear step, providing the vortex patches remain close to circular.

A pair initially translating in deep water with depth ratio $\gamma=H^{-} / H^{+}<1$, always crosses the escarpment and is refracted toward the normal to the step and cannot undergo TIR. The evolution of a vortex pair initially in shallow water, $\gamma>1$, depends on the size of the angle of incidence $\theta_{i}$ compared to the critical angle $\theta_{c}$ which is determined by the height of the escarpment and the initial separation of the pair (see (2.14)). If $\theta_{i}<\theta_{c}$, the pair refracts away from the normal. If $\theta_{i}=\theta_{c}$, the pair translates along the escarpment, and if $\theta_{i}>\theta_{c}$, the pair undergoes total internal reflection. 

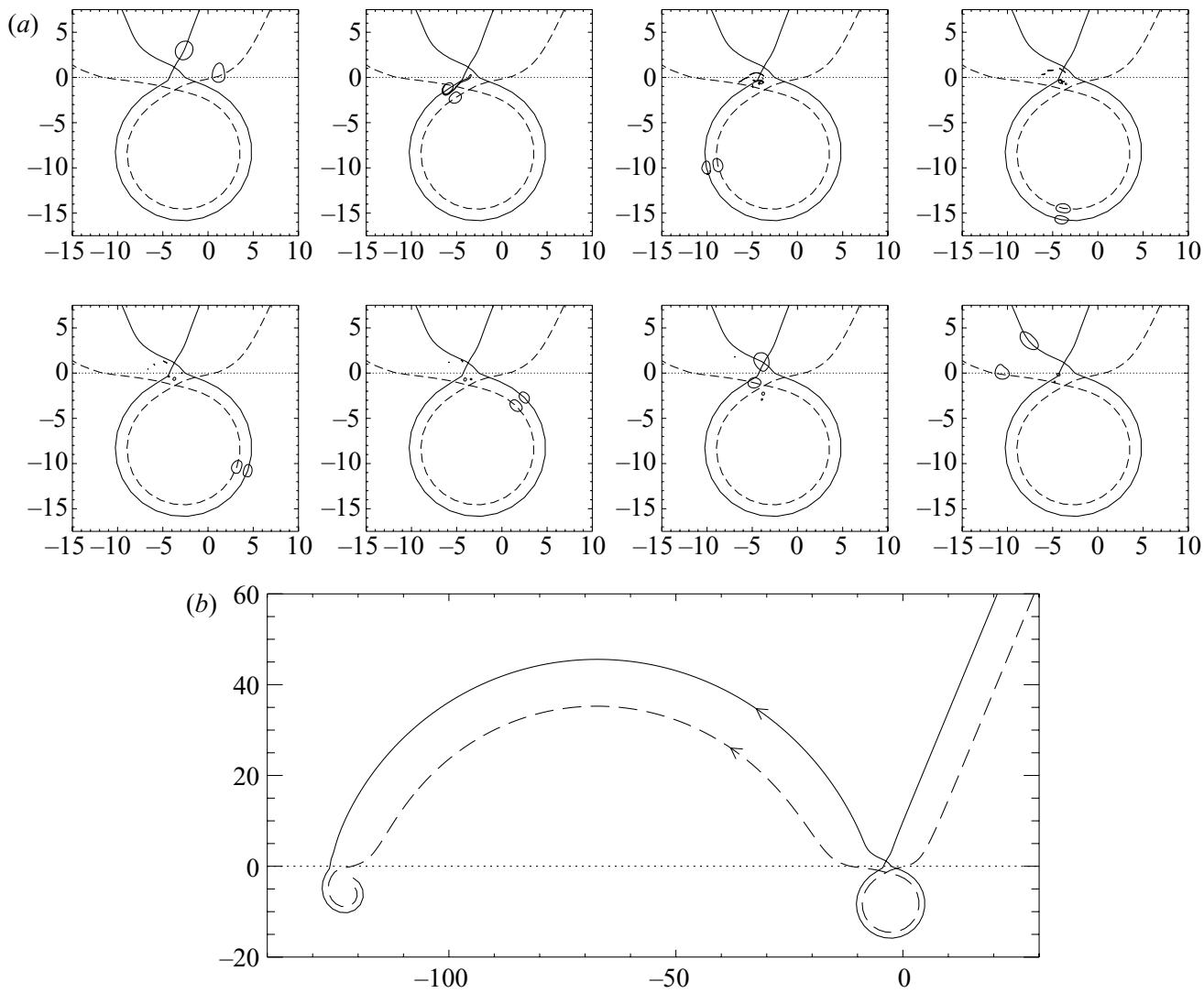

FIgURE 9. Trajectories and the evolution of a vortex pair with initial separation $\chi_{i}=7.5$. The large depth increase, $\gamma=2.5>2.4=\gamma_{d}$ means the trajectory lies in the debris region. $(a)$ Crossing and recrossing, $(b)$ the subsequent evolution over a much larger time.

If the depth ratio is greater than a critical value $\gamma_{d}$ (determined from (2.9)) then vorticity is shed by the patches when $\theta_{i}<\theta_{c}$, and subsequent paths are curved.

Numerical integrations of the full equations of motion supported the analysis with results for the angle of refraction agreeing closely with predictions provided vortex shedding was small. They also showed that for $\theta_{i} \gtrsim \theta_{c}$, it is possible for the pair to be captured at the escarpment and 'bounce' along the step.

The authors are indebted to Professor Dritschel for providing a copy of his 1988 contour surgery code which provided a basis for the computations. A. K. H. acknowledges support from a UK Engineering and Physical Sciences Research Council graduate training award.

\section{REFERENCES}

BüHLER, O. \& JACOBSON, T. E. 2001 Wave-driven currents and vortex dynamcis on barred beaches. J. Fluid Mech. 449, 313-339.

Chen, Q., Dalrymple, R. A., Kirby, J. T., Kennedy, A. B. \& Haller, M. C. 1999 Boussinesq modeling of a rip current system. J. Geophys. Res. 104, 20617-20637.

Dritschel, D. G. 1988 Contour surgery: a topological reconnection scheme for extended integrations using contour dynamics. J. Comput. Phys. 77, 240-266. 
Gorshrov, K. A., Ostrovsky, L. A. \& Soustova, I. A. 2000 Perturbation theory for Rankine vortices. J. Fluid Mech. 404, 1-25.

Johnson, E. R. \& McDonald, N. R. 2004 Surf zone vorticies over stepped topography. J. Fluid Mech. 511, 265-284.

Johnson, E. R., Hinds, A. K. \& McDonald, N. R. 2005 Steadily translating vortices near step topography. Phys. Fluids 17, 8.

Özkan-Haller, H. T. \& Kirby, J. T. 1999 Nonlinear evolution of the longshore current: a comparison of observations and computations. J. Geophys. Res. 104, 25 953-25984.

Peregrine, D. H. 1998 Surf zone currents. Theoret. Comput. Fluid. Dyn. 10, 295-309.

RichaRdson, G. 2000 Vortex motion in shallow water with varying bottom topography and zero Froude number. J. Fluid Mech. 411, 351-374.

Slinn, D. N., Allen, J. S., Newberger, P. A. \& Holman, R. A. 1998 Nonlinear shear instabilities of alongshore currents over barred beaches. J. Geophys. Res. 103, 18357.

Smith, J. A. \& LARGiER, J. L. 1995 Observations of nearshore circulation: Rip currents. J. Geophys. Res. 100, $10967-10975$.

YeH, P. 1998 Optical Waves in Layered Media. Wiley. 